\title{
Linx
}

Revue des linguistes de l'université Paris X Nanterre

$43 \mid 2000$

Linguistique de l'écrit, linguistique du texte

\section{Expolition Apotropaïque}

(en guise d'épiphtegme)

Jean-François Jeandillou

\section{OpenEdition}

Journals

Édition électronique

URL : http://journals.openedition.org/linx/1101

DOI : 10.4000/linx.1101

ISSN : 2118-9692

Éditeur

Presses universitaires de Paris Nanterre

Édition imprimée

Date de publication : 1 décembre 2000

Pagination : 159-165

ISSN : 0246-8743

Référence électronique

Jean-François Jeandillou, «Expolition Apotropaïque », Linx [En ligne], 43 | 2000, mis en ligne le 11 juillet 2012, consulté le 06 mai 2019. URL : http://journals.openedition.org/linx/1101 ; DOI : 10.4000/ $\operatorname{linx} .1101$

Ce document a été généré automatiquement le 6 mai 2019.

Département de Sciences du langage, Université Paris Ouest 


\title{
Expolition Apotropaïque
}

\author{
(en guise d'épiphtegme*)
}

Jean-François Jeandillou

Ainsi comprise comme principe de conciliation des
contraires et comme stratégie de simulation
énonciative, la déceptivité fait en sorte que le
discours n'embraye plus directement sur le réel et
prenne pour référent [...] un univers supplémentaire
à celui-ci ${ }^{1}$.

1 La question du texte reste, aujourd'hui plus que jamais, liée à l'autorité du discours dont il émane. A l'heure de la dématérialisation des "gros corpus » numérisés, au moment où les données virtuelles transitent de manière quasi instantanée au fil de réseaux planétaires, maintenant que la tchatche envahit les innombrables sites qui lui sont voués sur l'internet - «Entre ton pseudo! (six caractères au minimum, dix au plus)»-, se pose avec une insistance accrue le problème de l'auteur. Cette "fonction ", naguère théorisée (pour la mettre à bas) par Foucault, chacun est à même de la remplir lors d'échanges médiatisés par la machine plus encore que par le papier. Et ce qu'illustrent au mieux les nouveaux usages de l'écrit, c'est l'immarcescible disjonction entre une instance énonçante comprise (horresco referens) comme source productrice - et l'image (quot verba tot res) qu'en livre le texte. Le concept, si familier désormais, de pseudo veut bien dire ce qu'il dit, à savoir : "je mens, dans le temps même où j'inscris ce qui me sert de signature". Version moderne - en témoigne le grécisme apocopé - du fameux paradoxe d'Eubulide de Milet ${ }^{2}$ , cet acte de foi que détermine l'apposition du seing se retourne, inéluctablement, en profession de mauvaise foi. Tandis que le pseudonyme conserve quelque chose d'une unité onomastique - en quoi il est, précisément, mensonger -, le pseudo de nos Minitels et autres lambeaux de Toile est le pur signe performatif d'une dénégation, engagement à se démettre incontinent de toute responsabilité quant à la réception du texte ainsi ratifié. Sidérante façon de dire toute la vérité que de la dire en tiers, par une voie détournée qui, nonobstant, se signale comme telle ex abrupto ! Le mérite n'est pas aussi grand de ce nom, nécessairement emprunté, qu'est le patronyme : rien n'y garantit - l'auteur, on le sait, 
c'est le garant ${ }^{3}-$ la véridiction alors que tout l'abolit explicitement dans cette caution, sans conteste volontaire, qu'apporte le pseudo.

La question du texte reste, aujourd'hui plus que jamais, centrale dans les sciences du langage pour peu qu'elles affichent d'autres ambitions que celles, combien normatives, d'une grammaire de texte. Car l'enjeu n'est plus tant, maintenant, d'assurer la cohérence (logico-euphorique) du texte que d'assumer les incohérences (rhétorico-herméneutiques) destextes à travers leur diversité, leur banalisation, leur autorité débrayée. L'analyse discursive développée durant les dernières décennies a, souvent, fait du discours dit ordinaire un modèle de base pour l'analyse textuelle. D'où la fortune des «maximes conversationnelles » et autres "conditons de félicité » rapportées, au besoin pour en suspendre la pertinence, aux objets de langage les plus étrangers à tout souci de transparence et d'efficace. Mais le paradigme idyllique de la communication a fait son temps et l'on ne peut plus guère, désormais, postuler un degré zéro de la signification à partir duquel s'élaboreraient diverses pratiques plus ou moins compliquées, plus ou moins transgressives, plus ou moins saturées. Si la notion de cohésion et la présomption de cohérence, critères sine qua non des théories logicistes, ont connu un franc succès dans les champs de la sémantique vériconditionnelle et de la didactique, elles ont fourni DU discours une conception rationnellement séduisante mais régulièrement inapte à rendre compte de la variété DES textes. Or le linguiste, qui plus qu'au discours in abstracto a bel et bien affaire aux textes in situ, a tout intérêt à ne pas annihiler les éléments non cohésifs ou non cohérents dont il fait, comme lecteur d'abord, l'expérience. Un présupposé utile à la méthodologie heuristique ne saurait devenir constituant de l'objet qu'elle appréhende, sauf à fausser définitivement ce dernier. Autrement dit, la cohérence n'est jamais donnée d'avance, elle demeure tributaire d'une logique que chaque texte impose à l'herméneute en conférant aux mots leur valeur comme leur structure aux phrases. Ainsi, la langue apparaît moins un noyau dur autour de quoi évolueraient des usages ${ }^{4}$, qu'un principe dissipatif que les usages fixent de façon aussi provisoire qu'illusoire. Telle est finalement la puissance du montage textuel qu'il est en mesure de refaire, en permanence, et la langue et la logique. Sinon, pas de diachronie mais une éternité... de la pensée unique.

3 A défaut de citer les enchaînements, remarquables de congruence, que présentent la scène VIII de la Cantatrice chauve ou le Bétrou de Julien Torma (lequel ne fut sans doute pas sans incidence sur l'édifiante tchatche des Smith et des Martin), contentons-nous de renvoyer à la récente démonstration menée par Lita Lundquist ${ }^{5}$ pour prouver qu'une suite aléatoire - mais néanmoins calculée soigneusement, à cette fin, par l'expérimentateur... - de phrases parfaitement grammaticales peut constituer un « nontexte " pour cause de succession désordonnée. Bien que trois des trente-trois sujets sondés (soit près de dix pour cent, ou cent pour mille) l'aient jugée satisfaisante, en tant que texte à « effet particulier ", ladite séquence se voit impitoyablement qualifiée de nontextepar l'analyste au nom des " cataphores et anaphores », des " constructeurs d'espaces mentaux» et des «opérateurs argumentatifs». Fait empirique dirimant, l'intuition majoritaire constitue un gage probant pour éliminer, par pétition de principe, tout dispositif perçu comme « inhabituel », "poétique, littéraire » ou encore révélateur de "pensées incohérentes ». Curieuse méthode, qui évacue de son domaine d'investigation (le factum textus) les documents les mieux attestés: d'être littéraire ou poétique ou délirant un écrit ne serait plus texte ${ }^{6}$, seul ayant droit à cette scientifique appellation celui que la doxa du moment trouve bien (c'est-à-dire banalement) construit. A l'encontre d'une telle exclusive, décelant sous l'expression texte inhabituel une coïncidentia 
oppositorum, on pourrait objecter que c'est la notion de non-texte qui a tout, sauf pour des « scientistes» (p. 73) impénitents, d'un adynaton.

Emanation d'une raisonnable rationalité, la représentation ainsi promue n'est rien d'autre que celle d'un discours sans inconscient (quelle plus belle manifestation du SurMoi, avouons-le, que les marqueurs dits de progression thématique ?). D'où ces nouveaux épisodes, à suivre çà et là, des sempiternelles aventures d'Astérisque et Linguistix : il suffit pour en produire à foison de poser comme non recevables des énoncés qui, si même ils ne sont pas encore attestés, ne demandent qu'à l'être. Impossible, nous dit-on par exemple, de faire un incipit de *Je m'éveillai en proie à cette angoisse parce qu'un "nom comme angoisse ne permet pas, en l'absence d'une relative, d'introduire par une désignation démonstrative un référent nouveau [...]. On ne peut désigner par un GN dém qu'un objet pourvu d'une délimitation propre ${ }^{7}$ ». Or un acte d'autorité, sous quelque nom d'auteur qu'il s'accomplisse, se caractérise précisément par la possibilité qu'il offre de choisir pour incipit cet énoncé-là, tout contestable qu'il paraisse hors contexte ${ }^{8}$. La linguistique n'a pas à forger des modèles textuels conformes à l'idée qu'elle veut se faire de l'acceptabilité. Il lui revient de porter au jour des règles contingentes qui, soumises à des impératifs de genre eux-mêmes fluctuants et toujours innovants, se saisissent mal à coup de préceptes catégoriques (un texte peut / ne peut pas, doit / ne doit pas, etc.).

5 La question du texte reste, aujourd'hui plus que jamais, subordonnée à l'aperception de formes phrastiques sans guère de rapport avec les prescriptions élaborées par une syntaxe dont l'objet demeure, irrémédiablement, spéculatif. Aussi la stylistique, longtemps bannie des approches techniciennes, refait-elle surface et tend même à devenir comme un pôle fédérateur s'opposant, à divers titres, aux modélisations fixistes élaborées par le structuralisme et/ou le générativisme des années soixante. Au vu des travaux représentatifs de ces tendances (ré)novatrices, on peut néanmoins s'étonner du retour, souvent insidieux et le cas échéant dénégatif, de la notion d'écart, contre laquelle s'est naguère construite toute la description linguistique des textes. Comme si le fait de style ne se pouvait évaluer qu'à distance d'une norme qu'on croit être fait de langue, lui qui est avant tout une approche, asymptotique dans le meilleur des cas, du système linguistique et de sa productivité. Il ne suffit certes pas d'affirmer, à la suite de poéticiens comme Genette, que " tout texte a "du style" ${ }^{\text {" }}$ " pour redorer le blason de LA stylistique ; il ne suffit pas non plus, à la suite de théoriciens comme Compagnon, d'alléguer que «le pastiche prouve le style ${ }^{10}$ " pour concevoir le style en question autrement qu'à la manière d'une variation formelle sur un contenu prétendument stable. On le sait depuis les expérimentations de Pierre Ménard mises en lumière par Borges, le style peut différer du tout au tout quand même la lettre d'un texte est rigoureusement identique à celle d'un autre. L'enjeu n'est donc pas mince, pour la linguistique non dogmatique et pour les sciences du langage plus largement, de reconsidérer cette dimension du texte en prenant comme critère a minima des variations sémantiques que ne soutient aucune modification formelle. Sans doute le style s'en trouvera-t-il déplacé, ou replacé, du côté de la lecture interprétative plutôt que de la production; et le si suspect critère de l'écart, de n'être plus ponctuel ni déviant mais maximal et généralisé, y gagnera enfin, comme par usucapion, un fondement scientifique. Il n'y a pas plus d'objectivité du style que du texte, parce que l'un comme l'autre subissent de constantes mutations à travers le temps; tout est affaire, là encore, de diachronie autant que de représentations énonciatives et cognitives. 
6 L'autorité, la cohérence disruptive, le " stil ${ }^{11}$ ", voilà bien l'axe - Un en ceci même qu'il est trin - autour duquel gravitent chacune à leur manière les recherches ressortissant, dans ce volume, à la linguistique textuelle. Qu'ils aient - mais l'homme (qui se surnomma volontiers " Dédalus ») du Cymbalum est-il bien celui des Nouvelles Récréations? - ou non un auteur autre qu'anonyme, les textes renaissants que scrute Alexandra FLOIRAT se signalent par une polyphonie où l'on peut déceler les « indices » d'un discours indirect libre trop hâtivement jugé absent du XvI ${ }^{\mathrm{e}}$ siècle. Le problème du point de vue, de la légitimité de la parole et de son incidence sur la lisibilité, est traité là via le repérage de signaux, parfois hypothétiques mais souvent peu contestables, qui fondent en droit une étude grammaticale, co-textuelle et énonciative - où l'on ne s'étonnera pas de voir pointer l'ironie - de monuments plus modernes et précurseurs qu'on ne l'eût cru.

7 Linguiste du texte écrit s'il en fut, André ESKÉNAZI recense, dans l'éditionde 1674des Fourberies de Scapin, les fluctuations graphématiques affectant les initiales de mots. Au gré de leur distribution saisie en contexte local et structurée en paradigmes différentiels, les lettres tantôt capitales tantôt minuscules manifestent de subtiles variables sémantiques dont la stéréotypie même constitue un précieux indice des représentations culturelles. Plus que la norme d'un genre textuel, c'est en effet l'impensé de la langue classique ou, mieux, l'idéologie d'une époque qui transparaît ici de manière à la fois implicite (dans la mesure où, lors de la représentation théâtrale, le signifiant oral occulte le phénomène) et tangible à la lecture.

8 En prolongement de ses travaux, si novateurs et salutaires, sur la construction textuelle du point de vue, Alain RABATEL analyse par le menu les marques déceptives témoignant, plus que d'un brouillage indécidable, d'une attribution conflictuelle des jugements formulés soit par le narrateur (cette sorte d'auctor diégétique) soit par le personnage (autre sorte d'auctor, intradiégétique quant à lui). Il n'est d'ailleurs pas indifférent que le nom propre, pour fictionnel qu'il soit, serve en l'occurrence de gage à l'identification, sinon à la véridiction, dans la mesure même où il s'agit toujours d'un signe originaire qui confère à la parole son ancrage, fût-ce dans l'imaginaire. Et qu'est-ce au juste que le texte, sub specie lectionis, à part un vaste champ de «belligérance entre le savoir et le percevoir »?

9 Spécialiste des référents évolutifs, ces objets de discours presque aussi fuyants qu'un auteur à pseudo, Guy ACHARD-BAYLE pointe ensuite leurs modes de désignation pour montrer que l'effet de continuité, fait de texte à maints égards, est largement imputable à la coopération de l'interprétant - «nos vieilles habitudes de lecture » - et, au premier chef, à la croyance en la pérennité de ce que visent les mots. De la vérité de l'illusion à l'illusion de la vérité, il n'y a qu'un pas; sans se risquer à le franchir, on admettra néanmoins qu'il n'est point de cohérence sans la décison d'un lecteur bénévole, ni d'incohérence sans les réquisits d'un savant austère.

Enfin, fort des statistiques que lui fournissent les logiciels qu'il exploite - et perfectionne - au GRELIS de Besançon, Jean-Marie VIPREY présente une fine approche du matériel lexical des Fleurs du mal. Constitutives d'une véritable "cartographie hypertexte ", les récurrences et, surtout, les cooccurrences de vocables ou de formes linguistiques favorisent une approche stylistique des «isotropies », qui, outrepassant assurément la conscience qu'en avait le simple fils Dufaÿs, donnent toutefois une juste image de l'auteur Baudelaire. Par-delà toute apologie béate de l'énonciateur réel - seule instance de validation assignable et, comme le notait Foucault, «punissable»-, ce qui importe à 
l'analyse est moins l'illusoire identité (de re) d'un locuteurque l'ipséité (de dicto) d'un sujet irrémédiablement altéré, et ce faisant constitué, par le texte même.

11 Rien ne nous assure, au demeurant, que l'article signé par tel de nos ci-devant collaborateurs ne fut pas, horribile confessu, écrit par tel autre. Perspective au plus haut point fascinante - c'est-à-dire révoltante, aux yeux d'un agélaste inscient - que le logothète de Buenos-Aires ouvrit dès longtemps, en proposant de «parcourir l'Odyssée comme si elle était postérieure à l'Enéide » et d'«attribuer l'Imitation de Jésus-Christ à Louis-Ferdinand Céline ou à James Joyce ${ }^{12}$ ".

Si tel était ici le cas, la déontologie universitaire et l'honorabilité scientifique s'en trouveraient-elles meurtries? L'affaire Sokal, pour ne citer qu'elle, a montré que non: l'autorité pithanurgique y servit au contraire de caution, pour séduire les censeurs, à un canular qui n'était tel que par mimétisme envers ce qu'il moquait ${ }^{13}$. Peu importe la responsabilité de propos que le signataire tenait avec l'exacte intention de ne surtout pas les prendre à son compte ; le texte se charge assez, en pareille circonstance, de délester ce référent-père originel pour faire peser ladite responsabilité sur les lecteurs, qui doivent assumer seuls ce qu'il redoutent (et refusent) de comprendre. Car la cuistrerie ne se situe pas au principe de l'outrecuidance ; celle-ci résulte, tout entière, d'un mal entendu.

\section{NOTES}

* Ce texte reprend quelques éléments de la synthèse initialement proposée, sous le titre «Anacéphaléose », pour la Journée scientifique de l'EA 372 du 25 mai 2000. La composante TEXTE de l'UPRES entendait ainsi contribuer à un bilan quadriennal - «Résultats récents et questionnements actifs : d'hier à demain » - tout à la fois prospectif et pluridisciplinaire.

1. Jean Wirtz, Métadicours et déceptivité, Berne, Peter Lang, 1996, p. 136.

2. Bel exemple de corrélation entre le texte et l'autorité du discours, les paralogismes aporétiques du Crétois sont habituellement attribués à son compatriote Épiménide, qui aurait vécu quelque trois siècles après lui (Cf. l'article "sui-falsificateur" de B. Godart-Wendling, in les Notions philosophiques, Paris, Puf, 1990, t. 2, p. 2495).

3. Sur cette question, on peut lire le stimulant essai de P. Audi, l'Autorité de la pensée, Paris, Puf, 1997, notamment chap. 1 .

4. Comme l'illustrent clairement les schématisations proposées par J.-M. Adam in le Style dans la langue (Lausanne, Delachaux et Niestlé, 1997, p. 33) et Linguistique textuelle (Paris, Nathan, 1999, p. 93).

5. «Le Factum textus :fait de grammaire, fait de linguistique ou fait de cognition », Langue française $\mathrm{n}^{\circ} 121$, février 1999, p. 57 sq.

6. Telle était aussi, mutatis mutandis, l'opinion d'un Renaud Matignon à l'époque des premières publications de Nathalie Sarraute.

7. M.-N. Gary-Prieur, « La dimension cataphorique du démonstratif », Langue française $\mathrm{n}^{\circ} 120$, déc. 1998, p. 48 sq. D'après l'auteur, ladite délimitation serait, par compensation, palliée au moyen d'une relative restrictive: "Je m'éveillai en proie à cette angoisse que laissent derrière eux certains cauchemars nocturnes... " 
8. «Je m'éveillai en proie à cette angoisse. Je me levai, descendis à la cuisine pour y boire un verre d'eau. Encore cette angoisse... J'allumai la radio. Cette angoisse, toujours... Un bain tiède, quelques pas dans le jardin, une cigarette, rien n'y faisait: cette angoisse ne me lachait pas ", etc. Auster? Modiano? Echenoz? Toussaint? Let's guess!

9. Fiction et Diction, Paris, Éditions du Seuil, coll. « Poétique », 1991, p. 135.

10. Le Démon de la théorie, Paris, Éditions du Seuil, coll. « La couleur des idées », 1998, p. 202.

11. Ce " bipède au cou très long qui hante les autobus de la ligne $S$ vers midi », selon Queneau ( Exercices de style, Paris, Gallimard, 1947, p. 144.

12. Borges, «Pierre Ménard, auteur du Quichotte» (1939), in Fictions (1944), trad. fr. Paris, Gallimard, «Bibliothèque de La Pléiade », t. I, 1993, p. 475.

13. Pour un état récent de la question, articulé avec le thème «Herméneutique et sciences ", cf. le sévère trilogue entre P. Dumesnil, F. Rastier et J.-M. Salanskis, sur le site http://www.mshparis.fr/texto.

\section{AUTEUR}

JEAN-FRANÇOIS JEANDILLOU 